\title{
Functional annotation of the human brain methylome identifies tissue-specific epigenetic variation across brain and blood
}

\author{
Matthew N Davies ${ }^{1,2}$, Manuela Volta', Ruth Pidsley', Katie Lunnon', Abhishek Dixit', Simon Lovestone', \\ Cristian Coarfa ${ }^{3}$, R Alan Harris ${ }^{3}$, Aleksandar Milosavljevic ${ }^{3}$, Claire Troakes', Safa Al-Sarraj ${ }^{1}$, Richard Dobson? \\ Leonard C Schalkwyk ${ }^{1}$ and Jonathan Mill ${ }^{*}$
}

\begin{abstract}
Background: Dynamic changes to the epigenome play a critical role in establishing and maintaining cellular phenotype during differentiation, but little is known about the normal methylomic differences that occur between functionally distinct areas of the brain. We characterized intra- and inter-individual methylomic variation across whole blood and multiple regions of the brain from multiple donors.

Results: Distinct tissue-specific patterns of DNA methylation were identified, with a highly significant overrepresentation of tissue-specific differentially methylated regions (TS-DMRs) observed at intragenic CpG islands and low CG density promoters. A large proportion of TS-DMRs were located near genes that are differentially expressed across brain regions. TS-DMRs were significantly enriched near genes involved in functional pathways related to neurodevelopment and neuronal differentiation, including BDNF, BMP4, CACNA1A, CACA1AF, EOMES, NGFR, NUMBL, PCDH9, SLIT1, SLITRK1 and SHANK3. Although between-tissue variation in DNA methylation was found to greatly exceed between-individual differences within any one tissue, we found that some inter-individual variation was reflected across brain and blood, indicating that peripheral tissues may have some utility in epidemiological studies of complex neurobiological phenotypes.
\end{abstract}

Conclusions: This study reinforces the importance of DNA methylation in regulating cellular phenotype across tissues, and highlights genomic patterns of epigenetic variation across functionally distinct regions of the brain, providing a resource for the epigenetics and neuroscience research communities.

\section{Background}

DNA methylation is a key epigenetic mechanism involved in the developmental regulation of gene expression [1], but the tissue-specific nature of DNA methylation has not been fully characterized at a genomic level. Epigenetic processes control several neurobiological and cognitive processes, including neurogenesis and brain development [2], neuronal activity [3], learning and memory [4], drug addiction [5], neurodegeneration [6], and circadian rhythm [7]. The importance of DNA methylation in normal brain function and development is exemplified by the neurodevelopmental deficits

\footnotetext{
* Correspondence: jonathan.mill@kcl.ac.uk

'Institute of Psychiatry, King's College London, De Crespigny Park, London, SE5 8AF, UK

Full list of author information is available at the end of the article
}

associated with mutations in the methyl CpG binding protein 2 gene (MECP2) in Rett syndrome [8], and the aberrant DNA methylation signatures observed in neuropsychiatric disorders, including schizophrenia and bipolar disorder [9]. Although gene expression analyses have highlighted clear transcriptomic differences across brain regions [10-12], current studies of tissue-specific DNA methylation in the brain have assessed only a small percentage of $\mathrm{CpG}$ sites in the human genome [13], and none has taken an unbiased methylome-wide approach across multiple brain regions and blood obtained from the same individuals. Little is known, therefore, about normal methylomic differences between functionally distinct areas of the brain and how these correspond to patterns observed in easily accessible peripheral tissues such as blood. In this study we used
C Biomed Central

(c) 2012 Davies et al.; licensee BioMed Central Ltd. This is an open access article distributed under the terms of the Creative Commons Attribution License (http://creativecommons.org/licenses/by/2.0), which permits unrestricted use, distribution, and reproduction in any medium, provided the original work is properly cited. 
methylated DNA immunoprecipitation combined with ultra-deep sequencing (MeDIP-seq) to profile the methylomic landscape across multiple dissected brain regions and blood obtained from multiple individuals. We present annotated maps of the brain methylome, representing a unique resource for the genomics and neuroscience research communities, identifying key regions of the genome characterized by functionally relevant tissue-specific DNA methylation.

\section{Results and discussion}

\section{Methylomic profiling across brain and blood}

Our primary methylomic profiling experiments used multiple dissected brain regions (inferior frontal gyrus, middle frontal gyrus, entorhinal cortex, superior temporal gyrus of the temporal cortex, visual cortex, and cerebellum) from post-mortem brain samples obtained from individuals free of any neuropathology and neuropsychiatric disease. From a subset of these individuals, whole blood samples were also obtained longitudinally prior to death. A detailed list of the primary samples used in this study is given in Supplementary Table 1 in Additional file 1 . Of these, 21 tissue samples from three individuals (two female, one male) were initially assessed using ultra-deep pairedend MeDIP-seq (see Materials and methods). After stringent quality control (Supplementary Figure 1 in Additional file 1), an average of $>70.4$ million uniquely mapped $50 \mathrm{bp}$ reads were obtained from each of the 21 samples (Supplementary Table 2 in Additional file $1)$; to our knowledge, this represents the largest between-individual and cross-tissue DNA methylation dataset yet produced. To generate an estimate of actual DNA methylation from our MeDIP-seq data, we used the MEDIPS analysis package [14] to control for local CpG density and generate DNA methylation scores for overlapping $500 \mathrm{bp}$ bins across the genome. Bisulfite pyrosequencing was used to verify DNA methylation estimates at selected regions of the genome, and examine base pair-specific levels of DNA methylation across nominated regions in additional brain and blood samples. Normalized raw MeDIP-seq reads and MEDIPSestimated absolute DNA methylation values for each tissue/individual combination are available as a resource for download and browsing as UCSC tracks from our laboratory website [15]. The data are also being integrated into the Human Epigenome Atlas $[16,17]$ as part of the regular data release by NIH Epigenomics Roadmap Initiative [18].

Genome-wide DNA methylation across cortex, cerebellum and peripheral blood is highly tissue-specific

As expected, canonical genic DNA methylation profiles do not differ across samples or tissue types, with overall low average DNA methylation around the immediate transcription start site, high levels of DNA methylation across the gene body, and more subtle hypomethylation being observed at the 3' end of genes (Supplementary Figure 2 in Additional file 1), confirming previous observations [19]. Genome-wide, however, there are striking tissue-specific differences in DNA methylation, with a clear hierarchical distinction between the six cortical regions, cerebellum and blood (Figure 1a, b). These broad differences reflect the known developmental pathways of the three tissues; blood cells originate from the mesoderm, while cells of the central nervous system are ectodermic. Within the brain, the cerebellum develops from the metencephalon, whilst the cerebral cortex develops from the most anterior part of the neural plate (the telencephalon). The cortex itself is subdivided into numerous functionally distinct anatomical regions, specializing in sensory, motor, and association tasks.

\section{DNA methylation varies across different functionally annotated regions of the genome}

We defined feature sets spanning i) all annotated CpG islands (CGIs), sub-typed by location (gene promoter, intragenic, 3' UTR, and intergenic) as described previously [19], ii) CGI shores (spanning 2,000 bp up- and downstream of each CGI), and iii) all annotated coding sequences (CDSs). We also examined tissue-specific patterns of DNA methylation across gene promoters defined by low, medium and high CG content (LCPs, ICPs, and HCPs, respectively) [20]. BED files of the feature annotations used in this study (CGIs, CGI shores, CDSs, LCPs, ICPs, and HCPs) are available for download from our laboratory webpage [15]. DNA methylation across each feature was quantified using both normalized MeDIP-seq read counts and MEDIPS scores; Figure 2a shows the average DNA methylation levels for each feature type. As described previously [21], CGIs are significantly hypomethylated compared to CGI shores and the gene body, with no overall difference in canonical methylation patterns between tissues or individuals. There is, however, considerable heterogeneity in DNA methylation across different categories of CGI, dependent upon genomic location, with promoter CGIs being significantly hypomethylated in comparison to intragenic, 3' UTR, and intergenic CGIs. CGI shores, on the other hand, do not vary significantly as a function of genomic location. Although the majority of promoters (approximately 60\%) are associated with CGIs, not all promoter regions are hypomethylated; promoter methylation is inversely correlated with CG density, with LCPs being the most methylated of any feature type tested. In contrast, HCPs (which overlap considerably with promoter CGIs) are hypomethylated relative to the other features. 


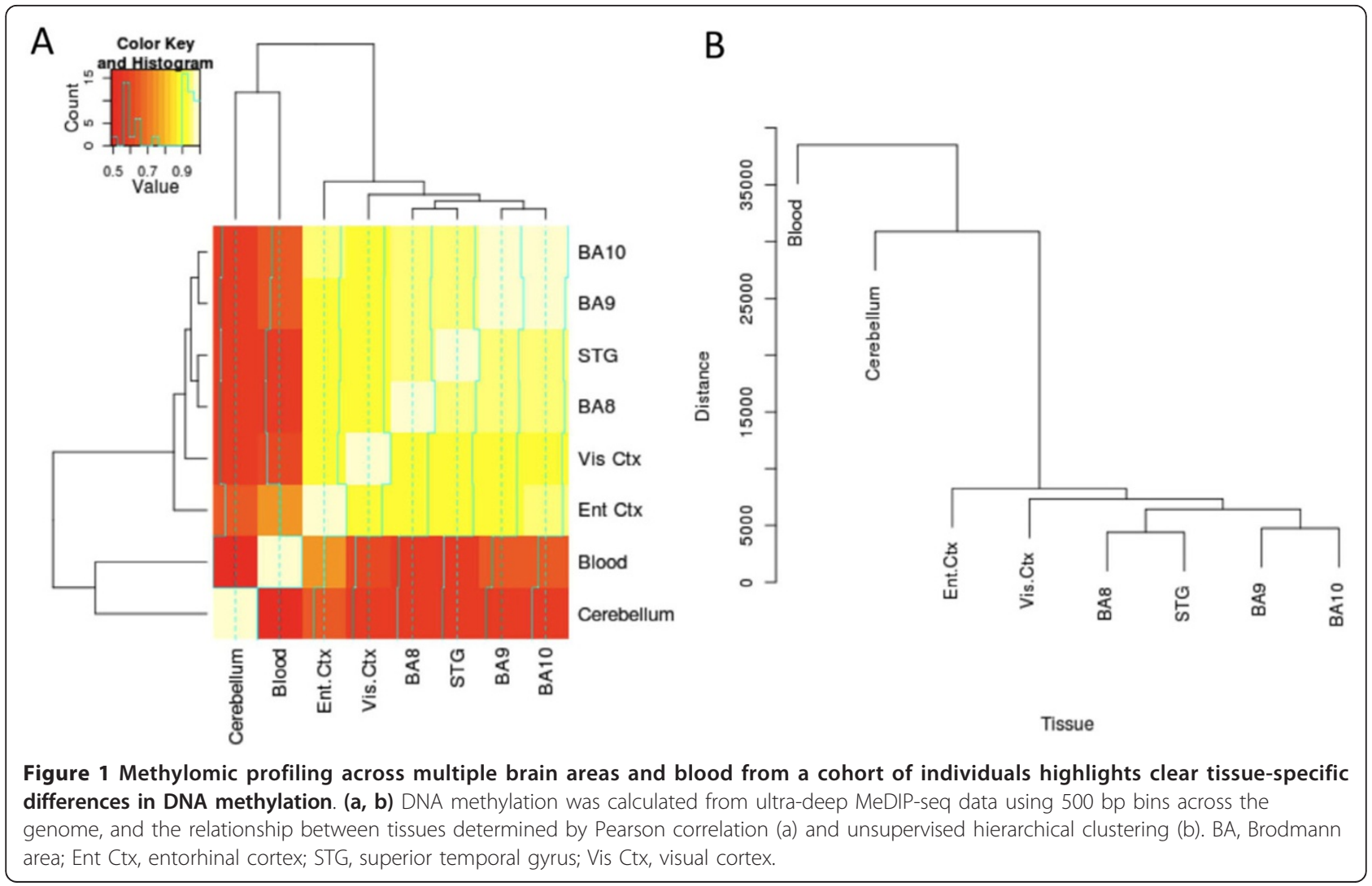

\section{DNA methylation differs significantly between tissues across all annotated feature types}

We next examined tissue- and brain region-specific DNA methylation in the context of annotated gene features to identify regions of the genome harboring tissue-specific differentially methylated regions (TSDMRs). Due to the overall similarity between the six cortical regions, we initially focused on gross differences between cortex, cerebellum and blood. Cross-tissue normalized MeDIP-seq data for all 65,535 annotated features can be downloaded from our laboratory website [15]. For each broad category of feature, hierarchical clustering of the MeDIP-seq data can clearly distinguish between tissue types (Supplementary Figure 3 in Additional file 1). The top 50 variably methylated annotated features across cortex, cerebellum and blood are listed in Supplementary Table 3 in Additional file 1. Using Illumina $450 \mathrm{~K}$ HumanMethylation microarray data obtained from matched cerebellum, frontal cortex, and whole blood samples from 90 individuals being assessed by our group as part of an ongoing clinical epigenetics study (Mill et al., unpublished data), we were able to confirm that the vast majority of these features are true TS-DMRs (Supplementary Table 4 in Additional file 1); 74\% of the 206 probes mapping to the broad genomic regions covered by these features were characterized by false discovery rate-significant intra-individual between-tissue DNA methylation differences in the direction predicted by our MeDIP-seq data.

Ingenuity Pathway Analysis (IPA) of the most variable features (defined as those with a coefficient of variance $(\mathrm{CV})>1$ across the three tissue types) highlights a highly significant enrichment of functional pathways involved in regulating developmental gene expression $\left(P=8.83 \times 10^{-25}\right)$, organismal development $\left(P=1.37 \times 10^{-20}\right)$, and tissue differentiation $(P=2.34$ $\left.\times 10^{-20}\right)$ (Supplementary Figure 4 in Additional file 1). Of note, given the origins of the samples used in this analysis, it is interesting that the primary tissue-specific functional pathways enriched in the list of TSDMRs are nervous system development and function $\left(P=4.60 \times 10^{-21}\right)$ and hematological system development and function $\left(P=3.61 \times 10^{-8}\right)$, indicating that epigenetic differences are likely to be associated with significant phenotypic differences. Although TS-DMRs are distributed across all feature types, there are marked differences in the between-tissue correlation of DNA methylation across each of the broad feature categories we examined, with CGIs being more correlated across cortex, cerebellum and blood than CGI shores or CDSs (Figure 2b). 


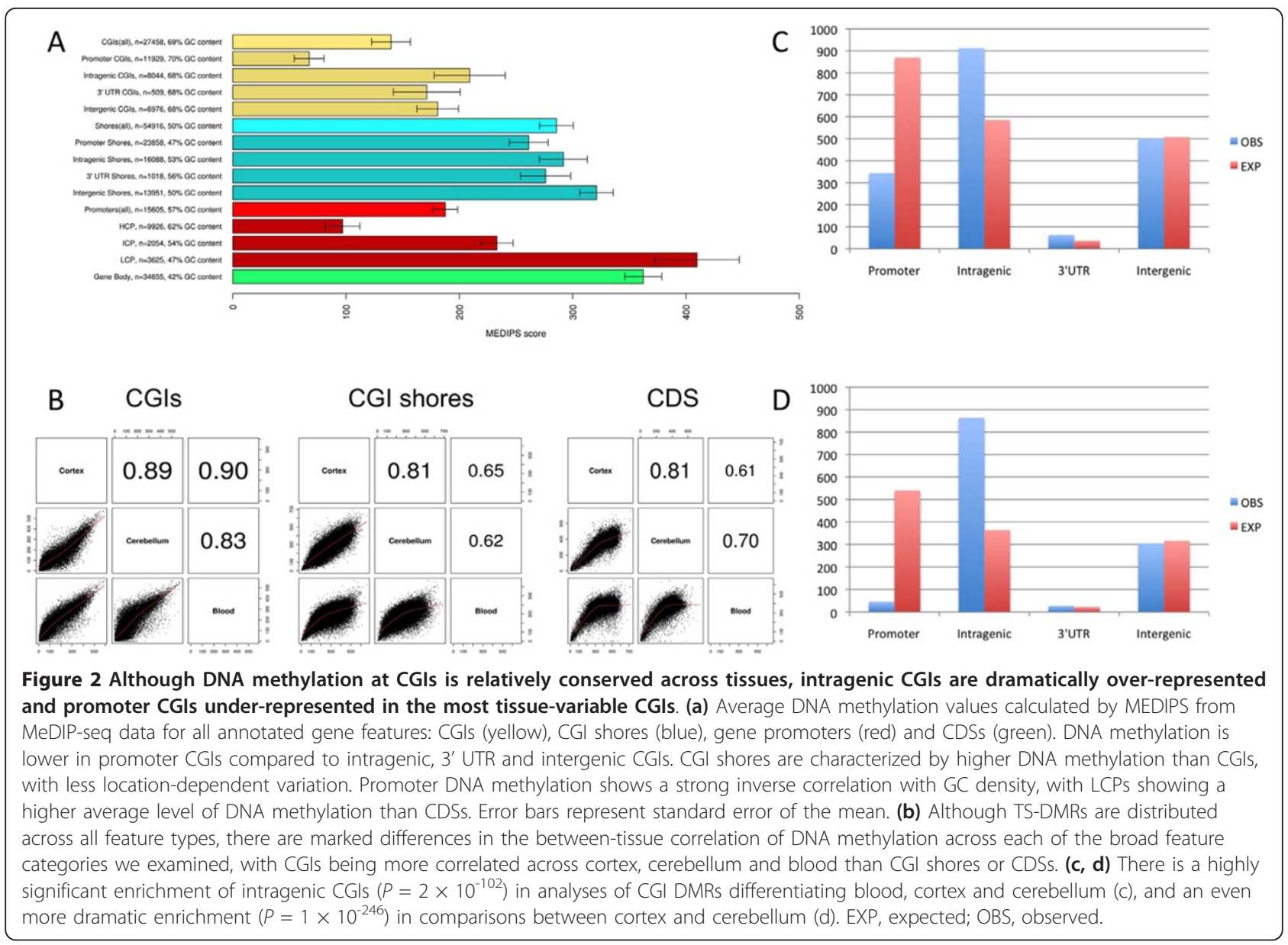

TS-DMRs across brain regions are associated with stable gene expression differences and are highly enriched for functionally relevant neurobiological pathways

Within the brain, the top 50 differentially methylated annotated features between cortical and cerebellum samples are listed in Supplementary Table 5 in Additional file 1. Strikingly, many of these DMRs are associated with genes of known relevance to cortical and/or cerebellar development and function, including $P P P 2 R 2 B$ (encoding a phosphatase implicated in the negative control of cell growth and division, whose disruption causes cerebellar ataxia [22]), JAKMIP1/MARLIN1 (encoding a RNA-binding protein that associates with GABA receptors [23]), EOMES (encoding a transcriptional activator that plays a crucial role in brain development, controlling the proliferation of intermediate progenitor cells and their progeny in the cerebral cortex [24]), GPMB6 (encoding a proteolipid widely expressed in neurons and in oligodendrocytes [25]), and GRM4 (encoding a metabotropic glutamate receptor with a distinct distribution in the brain, primarily expressed in cerebellar granule cells [26]). Furthermore, IPA of the top within-brain variable DMRs (differentiating cortex from cerebellum) highlights a primary network involved in nervous system development and function (Supplementary Figure 5 in Additional file 1 ), with highly significant enrichment of developmentally relevant pathways, including neurogenesis $(P=1.76$ $\left.\times 10^{-19}\right)$, the guidance of neurites $\left(P=2.11 \times 10^{-12}\right)$, development of the cerebellum $\left(P=8.49 \times 10^{-5}\right)$ and development of the cortex $\left(P=1.11 \times 10^{-4}\right)$ (Supplementary Table 6 in Additional file 1).

We next used RNA extracted from matched cerebellum and frontal cortex (Brodmann area (BA)9) samples, obtained from an independent cohort of 42 additional individuals, to assess tissue-specific expression levels for detectable gene transcripts located in the vicinity of the top 50 cortex-cerebellum DMRs. The majority (82\%) of these DMRs are mirrored by significant $(P<0.05)$ gene expression differences between cerebellum and frontal cortex (Supplementary Table 7 in Additional file 1), with $61 \%$ of genes being represented by at least one probe with a highly significant $\left(P<1 \times 10^{-10}\right)$ expression difference across the two brain regions. Interestingly, DNA methylation at these DMRs is not always negatively correlated with gene expression; EOMES, for 
example, shows highly significant elevated expression in the cerebellum compared to the frontal cortex $(\log 2$ expression values $=9.25$ versus $\left.6.46, P=1.90 \times 10^{-33}\right)$. An analysis of publically available gene expression data [12] confirms many of these gene expression differences and demonstrates that many of the cortex-cerebellum DMRs are associated with developmentally stable gene expression differences between the brain regions (Supplementary Figure 6 in Additional file 1). This further supports the notion that the TS-DMRs identified here mediate functionally important differences in the cellular transcriptome.

\section{DNA methylation differs across functionally discrete regions of the cerebral cortex}

Although for all features tested the six cortical regions formed a tight cluster, distinct from both cerebellum and blood (Figure 1a, b), we were interested to see if we could identify DMRs that could distinguish between them. Supplementary Table 8 in Additional file 1 lists the 50 most variably methylated features across samples obtained from the frontal cortex (BA8, BA9, and BA10), entorhinal cortex, superior temporal gyrus, and visual cortex. While the magnitude of within-cortex variation is clearly lower than observed between average cortex and either cerebellum or blood, there are some noticeable region-specific patterns of DNA methylation, particularly in the visual cortex. This list of DMRs contains a striking number of genes implicated in brain function related to the cortex and neurodevelopment, including CACNA1A and $C A C$ $N A 1 F$ (calcium-channel genes involved in neuronal growth and development and controlling the release of neurotransmitters [27]), GALNT9 (a brain-specific O-glycosylase [28]), SLC8A2/NCX2 (a sodium/calcium exchanger that has been shown to be important in synaptic plasticity and cortical development [29]), NUMBL (encoding a protein that maintains progenitor cells during cortical neurogenesis [30]), and GRIK5 (a receptor for the excitory neurotansmitter glutamate [31]). IPA on loci associated with the top 500 across-cortex variably methylated features highlights an interactive network of genes involved in neurodevelopment and function (Supplementary Figure 7 in Additional file 1), with a significant enrichment for functional pathways associated with neurogenesis and neuronal function, including many directly related to development of the cortex, such as 'forebrain development' $\left(P=6.15 \times 10^{-7}\right)$ (Supplementary Table 9 in Additional file 1).

\section{Although DNA methylation at promoter CGls is strongly conserved across brain areas and blood, tissue-specific} DNA methylation is particularly striking at intragenic CGls The top 50 differentially methylated CGIs between cortex, cerebellum and blood are listed in Supplementary
Table 10 in Additional file 1. Of particular interest is the observation that a CGI associated with $B M P 4$, which encodes a protein mediating differentiation of the ectoderm into the nervous plate [32], is one of the strongest CGI DMRs between blood and brain (cortex and cerebellum). Also showing highly variable patterns of DNA methylation between brain tissues and blood are CGIs associated with other key neurodevelopmental genes, including $B D N F$ (encoding a neurotrophic factor with an important role in neurodevelopment and neurogenesis [33]) and SLITRK1 (encoding an integral membrane protein involved in neurite outgrowth [34]). IPA of the most variable $(\mathrm{CV}>1)$ blood versus brain CGIs reveals a highly significant enrichment of functional pathways involved in basic tissue development, including loci regulating both neurogenesis $\left(P=9.88 \times 10^{-14}\right)$ and hematopoiesis $\left(P=4.59 \times 10^{-8}\right)$ (Supplementary Table 11 in Additional file 1). Bisulfite pyrosequencing was used to verify selected CGI DMRs, and examine base pair-specific levels of DNA methylation across the nominated regions in additional samples. These included the top differentially methylated CGI, located within the $J M J D 2 B / K D M 4 B$ gene, encoding a histone demethylase that specifically demethylates histone $\mathrm{H} 3$ lysine 9, and highly ranked DMRs associated with the neurodevelopmental genes $B D N F$ and EOMES. We observed a highly significant correlation between our MeDIP-seq and pyrosequencing data (correlation $=0.58, P=7.07 \times 10^{-13}$; Supplementary Figure 8 in Additional file 1), with highly significant between-tissue DNA methylation differences, confirming the MeDIP-seq data, being observed for all tested bisulfite-PCR amplicons (Figure 3).

We observed a highly significant $\left(\chi^{2} P\right.$-value $=2 \times 10^{-}$ ${ }^{102}$ ) over-representation (observed/expected $(\mathrm{o} / \mathrm{e})=1.73$ ) of intragenic CGIs amongst these TS-DMRs, with a complementary under-representation $(\mathrm{o} / \mathrm{e}=0.43)$ of promoter CGIs (Figure 2c) and a highly significant difference in average CV between these two classes of CGI (promoter CGI mean $\mathrm{CV}=0.35$, intragenic CGI mean $\left.\mathrm{CV}=0.60, P<1 \times 10^{-10}\right)$. Promoter CGIs are also significantly less-variable across tissues compared to 3 ' CGIs (mean CV $=0.59$ ) and intergenic CGIs (mean CV $=0.50$ ). A comparison of the most differentially methylated CGIs between cerebellum and cortex, which again are significantly enriched for pathways related to brain development, neurogenesis and functional specialization in the brain (Supplementary Table 12 in Additional file $1)$, highlights an even more dramatic over-representation of intragenic CGIs $(\mathrm{o} / \mathrm{e}=2.37)$ and under-representation of promoter CGIs $(\mathrm{o} / \mathrm{e}=0.09)\left(\chi^{2} P\right.$-value $=1 \times$ $10^{-246}$; Figure $2 \mathrm{~d}$ ). To explore the functional organization of DNA methylation at intragenic CGIs across cortex, cerebellum and blood, we used weighted gene comethylation network analysis [35] to identify modules of 


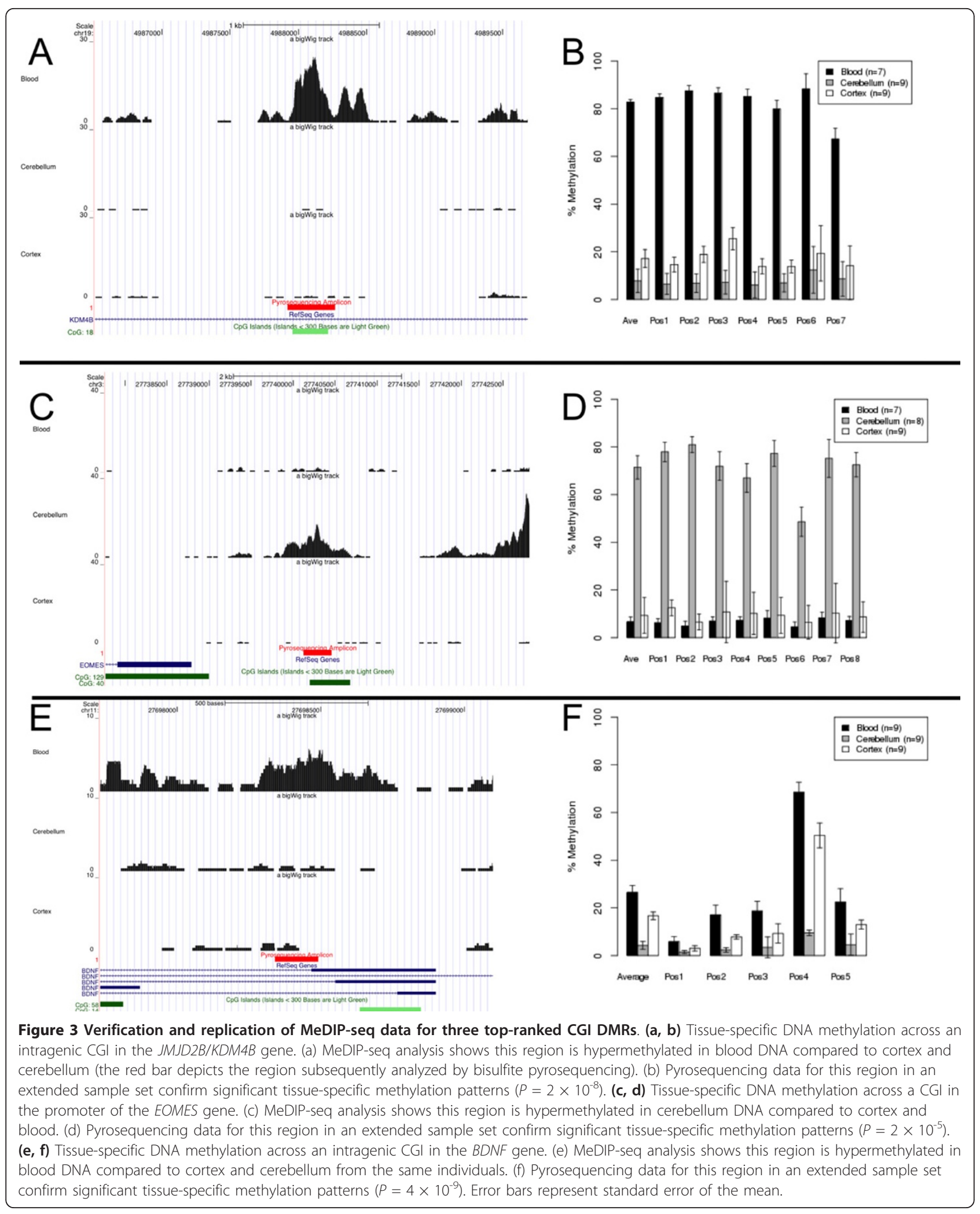


co-methylated features via unsupervised hierarchical clustering on the basis of high topological overlap (see Materials and methods). Six modules were identified with clear tissue-specific patterns, demonstrating that the methylome is organized into modules of co-methylated features (Figure 4a). The strongest tissue-specific module (blue), composed of approximately 1,000 intragenic CGIs that were hypomethylated in the cortex compared to cerebellum and blood (Pearson correlation $\left.=-0.98, P=4 \times 10^{-5}\right)$, representing a network of genes involved in nervous system development and function (Figure $4 \mathrm{~b}$ ) and significantly enriched for functional pathways, including neurogenesis $\left(P=2.47 \times 10^{-25}\right)$ and the differentiation of neurons $\left(P=6.18 \times 10^{-18}\right)$. Analysis of the top $5 \%$ of genes ranked by module membership using publically available gene expression datasets by GeneMania [36] showed that $58.9 \%$ are also coexpressed, indicating that the co-methylation networks identified here map onto functional gene coexpression networks. Furthermore, genes in this module are significantly enriched in relevant gene expression modules from published brain gene expression datasets (Supplementary Table 13 in Additional file 1). A role for differential DNA methylation at intragenic CGIs across different cell types in the mouse hematopoietic lineage has been recently reported [37], and it has been suggested that these features may regulate transcription from alternative promoters across specific cell types [19].

On average, CGI shores show more tissue-specific DNA methylation than CGls, but do not vary by genic location CGI shores have been previously shown to harbor phenotypically relevant tissue-specific patterns of DNA methylation [21]. Compared to CGIs, we found CGI shores to be significantly more variable across brain areas and blood (average CGI CV $=0.46$, average CGI shore $\mathrm{CV}=0.57, P<1 \times 10^{-10}$ ), with DNA methylation noticeably less correlated across tissues, especially between brain (cortex and cerebellum) and blood (Figure 2b). Unlike for CGIs, however, the location of CGI shores makes little impact on their variability across tissues with no over-representation of intragenically located features (Supplementary Figure 9 in Additional file 1). The top 50 differentially methylated CGI shores between cortex, cerebellum and blood are listed in Supplementary Table 14 in Additional file 1. Strikingly, many are associated with genes of known neurobiological function, including $P C D H 9$ (encoding a cadherinrelated neuronal receptor that localizes to synaptic junctions, involved in specific neuronal connections and signal transduction [38]), NGFR (encoding the receptor for nerve growth factor, with widespread effects on neurodevelopment [39]), AUTS2 (encoding the autism susceptibility candidate 2 , a nuclear protein expressed in developing brain regions [40]), SHANK3 (encoding a scaffold protein involved in the structural and functional organization of the post-synaptic density and also implicated in autism [41]), and SLIT1 (encoding a protein with a key role in cortical development and synaptogenesis [42]). IPA of the most variable CGI shores again reveals a highly significant enrichment for functional pathways involved in nervous system function and development (Supplementary Table 15 in Additional file 1). Bisulfite pyrosequencing was used to confirm and replicate the two top-ranked CGI shore DMRs, which are highly significantly hypermethylated specifically in the cerebellum, flanking a hypomethylated intergenic CGI on chromosome 7 (Supplementary Figure 1 in Additional file 10). Again, our pyrosequencing data were significantly correlated with the MEDIPS scores across each of the three amplicons spanning this region (left shore, correlation $=0.76, P=5.58 \times 10^{-5}$; CGI, correlation $=0.80, P=1.18 \times 10^{-5}$; right shore, correlation = $\left.0.82, P=4.36 \times 10^{-6}\right)$, confirming the validity of the methylome data.

\section{Low CG content promoters are also characterized by widespread tissue-specific DNA methylation across brain regions and blood}

Our CGI data concur with the notion that CpG-rich promoters (that is, HCPs) are predominantly hypomethylated and associated with ubiquitously expressed house-keeping genes $[20,43]$. We next decided to compare HCP methylation with that seen in LCPs; recent methylomic analyses of other tissues indicate that differential DNA methylation across LCPs is associated with tissue-specific gene expression in somatic cells [20]. Our data provide strong evidence to support this notion, and like intragenic CGIs, LCPs appear to be a major location for tissue-specific DNA methylation signatures across brain regions and blood. While hierarchical clustering of both HCP and LCP DNA methylation can distinguish between tissues, the Euclidian distance between tissues is much larger in the case of LCPs (Figure 5a). Principal components analysis of our MeDIP-seq data shows a much stronger tissue classification based upon LCP methylation (Figure $5 \mathrm{~b}$ ), and correlation analyses show that while HCP methylation is largely conserved across tissues (reflecting the pattern seen for promoter CGIs), the correlation of LCP methylation across tissues is much lower.

\section{Between-individual differences in DNA methylation are correlated across brain and blood}

Because aberrant DNA methylation is being increasingly implicated in the etiology of complex disease phenotypes, including several mental health disorders [44], a key 

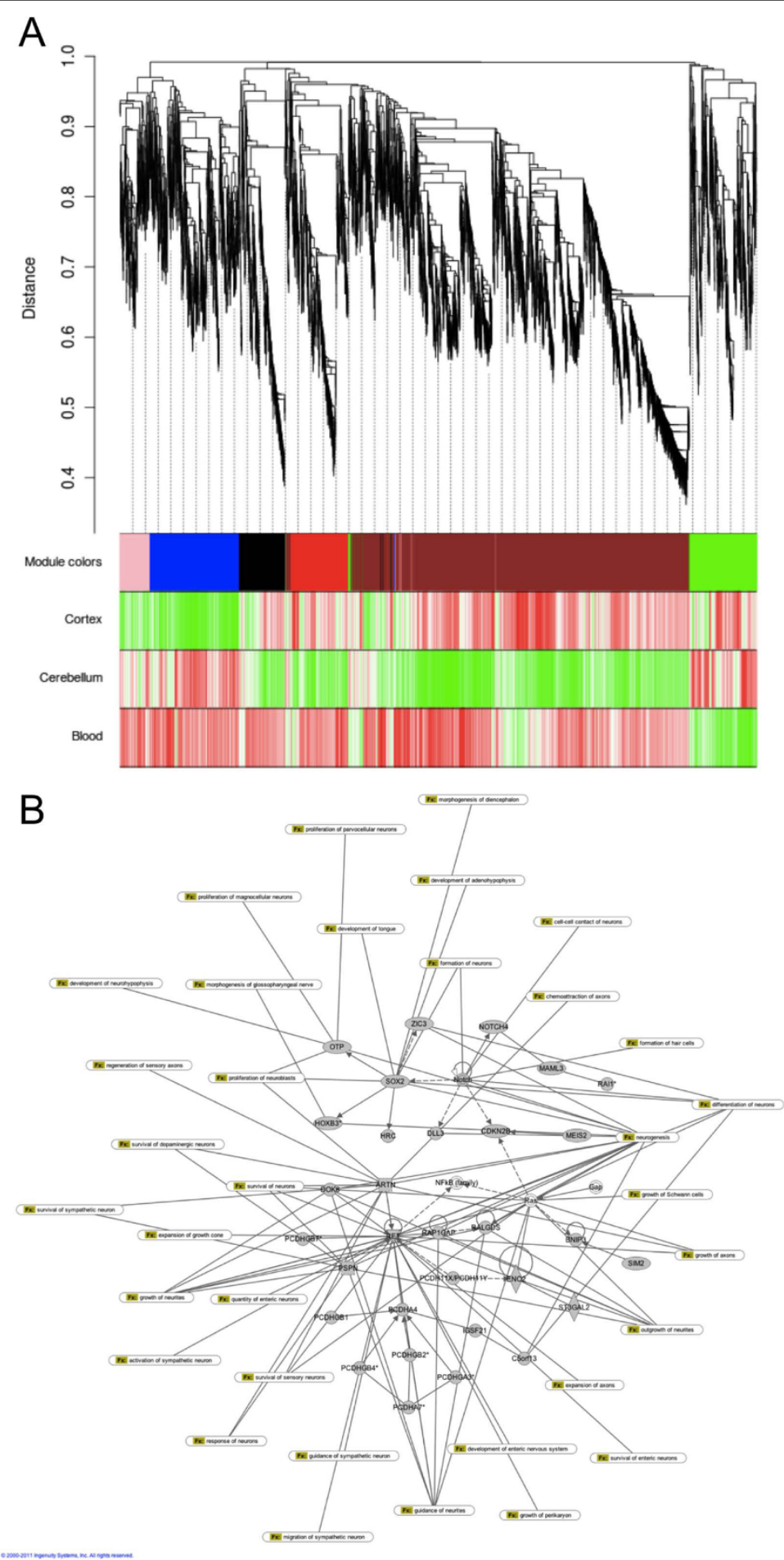

Figure 4 Weighted gene co-methylation network analysis of DNA methylation at intragenic CGls. (a) Dendrograms produced by average linkage hierarchical clustering of intragenic CGIs on the basis of topological overlap. Modules of co-methylated loci were assigned colors as indicated by the horizontal bar beneath each dendrogram. The 'blue' module was strongly negatively co-methylated $\left(r^{2}=-0.98, P=4 \times 10^{-5}\right)$ in cortex. (b) IPA on the genes associated with the blue module highlighted a network involved in nervous system development and function. 

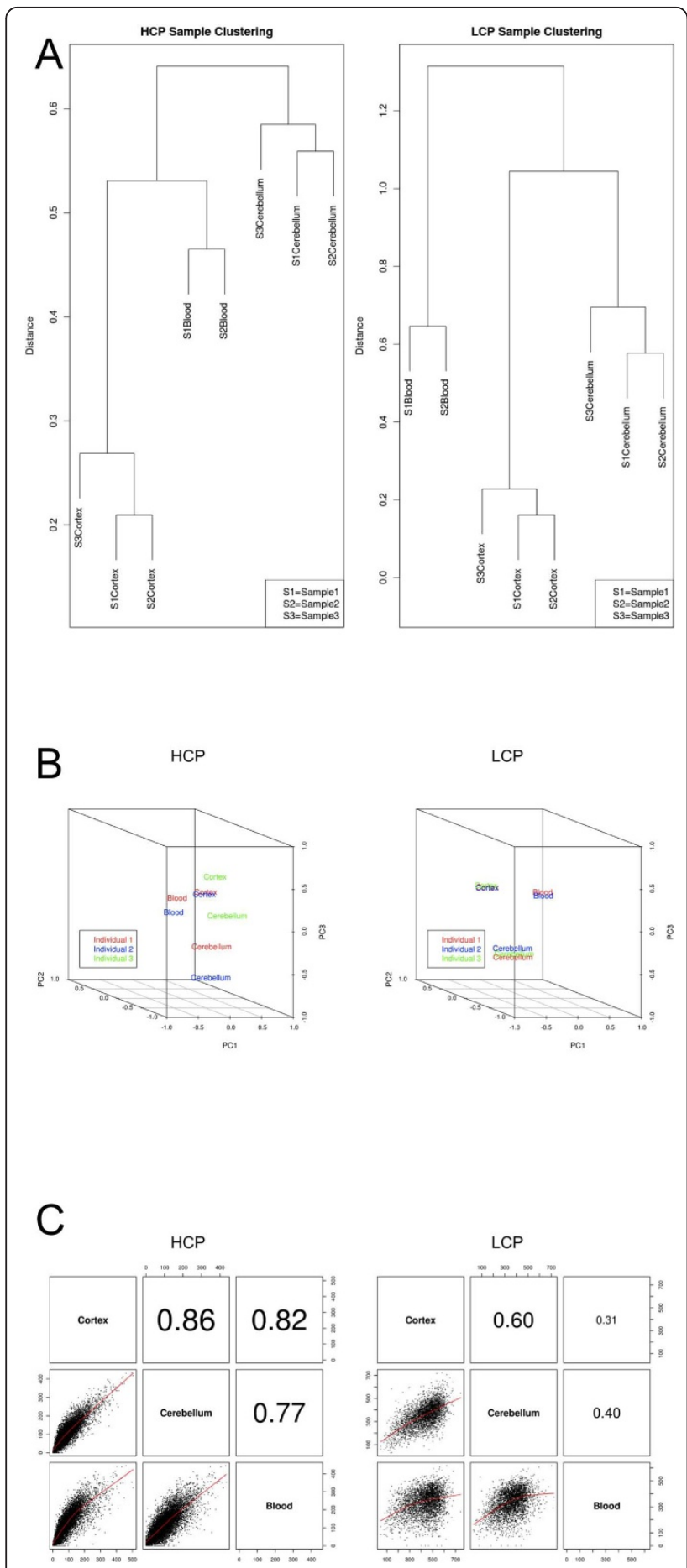

Figure 5 DNA methylation across LCPs is strongly associated with tissue type. (a) MEDIPS scores across both HCPs and LCPS can be used to accurately cluster samples by tissue type, but the strength of clustering, indicated by Pearson dissimilarity on the $y$ axis, is much higher in LCPs. (b, c) This pattern is reflected in threefactor PCA plots (b) and correlation analyses (c), with LCPs demonstrating stronger tissue-specific patterns of DNA methylation than HCPs. question in epigenetic epidemiology concerns the extent to which easily accessible peripheral tissues (for example, whole blood) can be used to ask questions about interindividual phenotypic variation manifest in inaccessible tissues such as the brain $[45,46]$. Our use of brain tissue and blood obtained pre-mortem from the same individuals enabled us to investigate the extent to which betweenindividual methylomic variation in the blood is reflected across the cortex and cerebellum. Comparing MeDIP-seq data from blood, cortex and cerebellum, we observe that inter-individual DNA methylation differences are highest in blood and lowest in the cortex, with a highly significant difference in variability across tissues $(P<0.001$; Figure $6 a)$. The pattern of relative inter-individual variability by feature is, however, the same across tissues, with non-promoter CGIs showing a significantly higher level of between-individual DNA methylation differences than other features. Strikingly, there is a significant correlation between individual DNA methylation differences in the blood and those in brain tissue (cortex and cerebellum) from the same two individuals (Figure 6b), with the most reproducible pattern of blood-detected individual differences observed in the cerebellum (correlation $=0.76, P<$ 0.001 ) and slightly less correlation between blood and cortex (correlation $=0.66, P<0.001)$. Although replicate DNA samples from each individual were assessed using high-resolution SNP arrays to identify potential structural variants and minimize the confounding effect of inter-individual copy-number variations ( $\mathrm{CNVs}$ ) that could manifest as MeDIP-seq read differences (see Materials and methods), the influence of genomic differences between individuals cannot be fully excluded. Supplementary Table 16 in Additional file 1 lists the top 50 between-individual differences in DNA methylation identified in blood between the two female MeDIP-seq blood samples and lists the corresponding methylation scores from cortex and cerebellum from the same individuals. Interestingly, while many features show near-identical between-individual patterns across all three tissues, some examples of blood-identified variation are only detected in either the cortex or cerebellum. Given recent reports by us and others of widespread genotype-associated allele-specific DNA methylation [47], it is likely that many of these between-individual DNA methylation differences are mediated by DNA sequence variation that is common across tissues, although other mechanisms such as epigenetic changes occurring early in development before complete tissue differentiation could also be important.

\section{Conclusions}

We used MeDIP-seq to undertake the first genomic characterization of intra- and inter-individual variation 


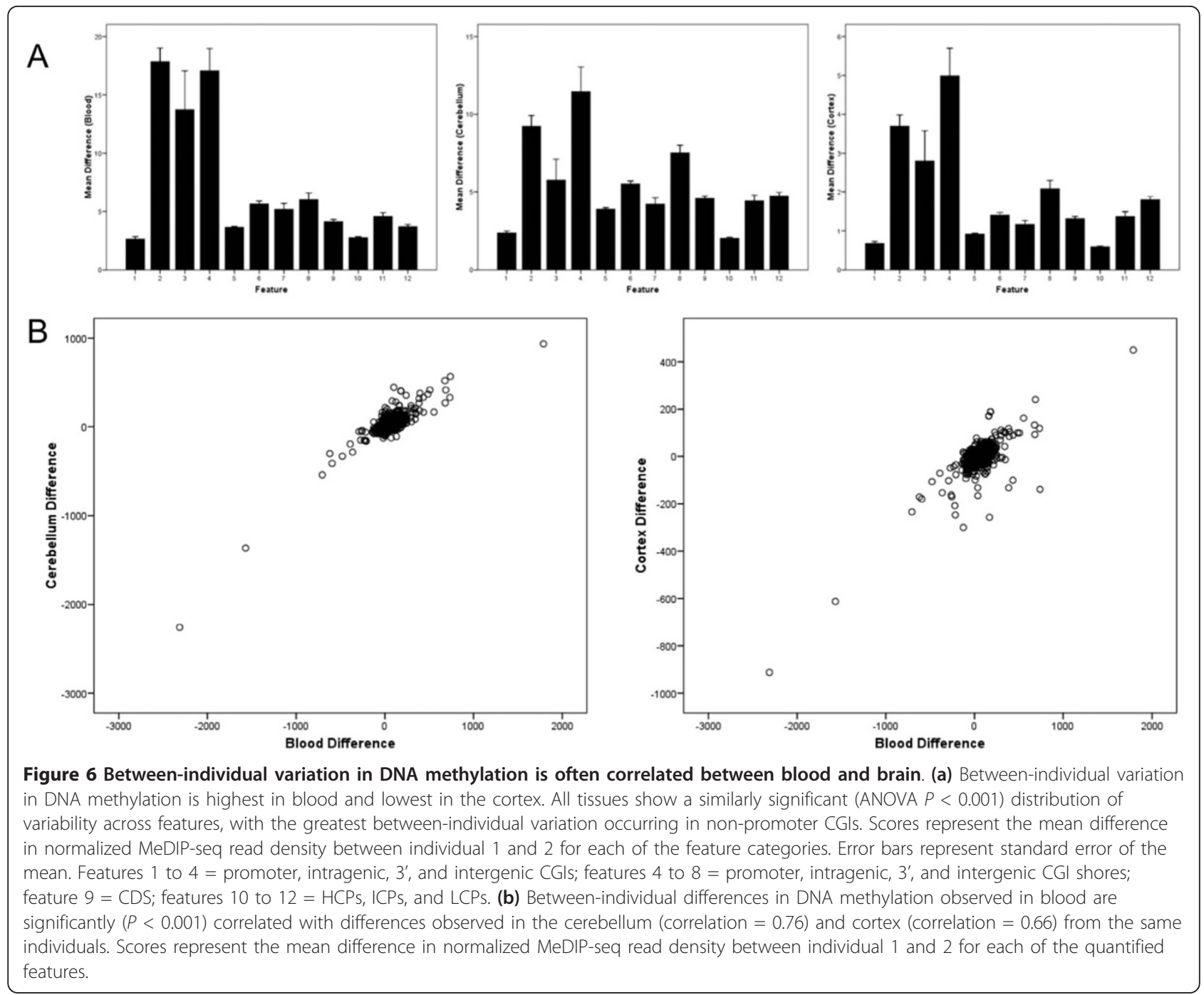

in DNA methylation across multiple regions of the human brain and an easily accessible peripheral tissue (whole blood). In summary, we show that between-tissue variation in DNA methylation greatly exceeds between-individual differences within any one tissue, with clear hierarchical differences in DNA methylation across specific brain areas, and between brain and blood. Interestingly, we observe that TS-DMRs are strikingly under-represented in classic promoter CGIs, being located primarily in intragenic CGIs and LCPs. These TS-DMRs are dramatically enriched for genes known to be involved in brain development and neurobiological function, forming networks of co-methylated loci that define the cellular phenotype. Finally, although inter-individual differences in DNA methylation are dwarfed by tissue-specific variation, and absolute levels of DNA methylation at specific loci clearly differs between cortex, cerebellum and blood, we observe that some between-individual variation in
DNA methylation is correlated between brain regions and blood.

To our knowledge, this study represents the most comprehensive cross-tissue inter-individual assessment of the methylomic landscape of the brain yet undertaken. The data generated in this project are available as a resource to the genomics research community; annotated UCSC tracks can be downloaded from our laboratory website [15], and the raw data are being integrated into the Human Epigenome Atlas [16] as part of the regular data release by NIH Epigenomics Roadmap Initiative. A primary goal of global research initiatives such as the International Human Epigenome Consortium and the NIH Epigenomics Roadmap is to create high-resolution reference epigenome maps across multiple human tissue and cell types that will expedite the application of epigenomic technologies to studies of human health and disease [18]. In this regard, and given increasing evidence supporting a role for epigenetic 
disruption in neuropsychiatric disease, a unique aspect of this study was our use of blood and multiple brain regions from the same individuals, enabling us to address questions about the extent to which easily accessible peripheral tissues can be used to ask questions about inter-individual phenotypic variation manifest in inaccessible tissues such as the brain.

In addition to creating reference maps of DNA methylation across multiple brain regions and blood, we have identified key regions of the genome that are characterized by tissue-specific patterns of DNA methylation. Of note, TS-DMRs were significantly enriched near genes involved in functional pathways related to neurodevelopment and neuronal differentiation, including $B D N F$, BMP4, CACNA1A, CACA1AF, EOMES, NGFR, NUMBL, PCDH9, SLIT1, SLITRK1, and SHANK3. Although DNA methylation at promoter CGIs is largely conserved across tissues, reflecting data from other studies, we find striking evidence that intragenic CGIs are a primary location for TS-DMRs. This builds on recent data highlighting cell type-specific DNA methylation at intragenic CGIs in the immune system [37], and evidence that these features may regulate transcription from alternative promoters across specific cell types [19]. Our data highlight LCPs as another region characterized by considerable cross-tissue epigenetic variation; again these data support recent methylomic analyses of other tissues, indicating that differential DNA methylation across LCPs is associated with tissue-specific gene expression in somatic cells [20]. Given the striking enrichment of tissue-relevant pathways and gene sets amongst the loci associated with the TS-DMRs we have identified, and the observation that many of these genes are differentially expressed across brain regions (Supplementary Table 7 and Supplementary Figure 6 in Additional file 1 ), it is likely that they mediate functionally relevant differences in the cellular transcriptome.

There are several limitations to this study. First, although it represents one of the largest cross-tissue methylomic analyses performed to date, the number of individuals profiled in our initial MeDIP-seq screen was relatively small. Our successful verification and replication experiments (using bisulfite pyrosequencing and the Illumina $450 \mathrm{~K}$ array), combined with our gene expression data, in larger number of samples, however, highlights the validity of the between-tissue differences we observe. Second, the samples used in our initial MeDIPseq screen were all obtained from donors $>75$ years old and may not fully represent patterns of DNA methylation earlier in life. However, our observation of highly significant gene expression differences corresponding to the top 50 cortex-cerebellum DMRs in a younger cohort of samples (average age $=62 \pm 18$ years) suggests that many of the tissue-specific differences observed are developmentally stable, resulting in functional differences at an earlier age. Future work should focus on identifying developmental trajectories of epigenetic change across multiple tissues.

In summary, this study reinforces the importance of DNA methylation in regulating cellular phenotype across tissues, and highlights genomic patterns of epigenetic variation across functionally distinct regions of the brain.

\section{Materials and methods}

\section{Sample preparation}

Post-mortem brain samples from nine elderly control individuals (free of neuropathological and neuropsychiatric disease) were obtained from the MRC London Neurodegenerative Diseases Brain Bank. Multiple brain regions were dissected from each sample by a trained neuropathologist, snap-frozen and stored at $-80^{\circ} \mathrm{C}$. Genomic DNA was isolated from each dissected brain region from each sample using a standard phenolchloroform extraction method, and tested for degradation and purity prior to analysis. From a subset of individuals whole blood samples were also obtained longitudinally prior to death, and DNA extracted using a standard phenol-chloroform method. A detailed list of the samples used for methylomic profiling in this study is given in Supplementary Table 1 in Additional file 1. An independent set of matched frontal cortex and cerebellum samples (average age $=62 \pm 18$ years, 33\% female) for gene expression analysis was obtained from 42 additional individuals provided by the London Neurodegenerative Diseases Brain Bank.

\section{Methylated DNA immunoprecipitation and sequencing}

DNA was fragmented using a Covaris sonication system and sequencing libraries were prepared from $5 \mu \mathrm{g}$ fragmented genomic DNA. End repair, $<$ A $>$ base addition and adaptor ligation steps were performed using Illumina's Paired-End DNA Sample Prep kit. Adaptor-ligated DNA was immunoprecipitated by anti-5mC using a commercial antibody (Diagenode, Liège, Belgium), and MeDIP products were validated by quantitative PCR. MeDIP DNA was purified with ZYMO DNA Clean \& Concentrator- 5 columns, and amplified using adaptormediated PCR. DNA fragments between 220 and 320 bp in size were gel-excised, and amplification quality and quantity were evaluated by Agilent BioAnalyzer analysis. The libraries were subjected to highly parallel $50 \mathrm{bp}$ paired-end sequencing on the Illumina Hi-Seq platform.

\section{Sequencing quality control and alignment}

From the raw fastq files, Illumina quality scores were converted into Sanger Phred quality scores using MAQ [48]. Quality control was performed on the raw 
sequence data using FastQC [49]. Supplementary Figure 1 in Additional file 1 shows FastQC output for one representative sample highlighting the high quality sequencing data obtained. Alignment to hg18 was performed using the Burrows-Wheeler algorithm.

\section{Correcting for local CpG densities}

The MEDIPS package [14] was used to calculate methylation scores by incorporating a coupling factor based on local CpG density. Bin sizes of 500 bp were defined across the genome, with an overlap of $250 \mathrm{bp}$. The number of CpGs within the maximal defined distance around the genomic bin was calculated and a calibration curve determined relative to the dependency of local MeDIP-seq signal intensities and local CpG densities.

\section{DMR calling across known features using normalized read counts}

Mapped reads were also quantified using SeqMonk (Babraham Institute, Cambridge, UK). BED files of the feature annotations used in this study (CGIs, CGI shores, CDS, LCPs, ICPs, and HCPs) are available for download from our laboratory webpage [15]. Readdepth scores were generated for each feature, normalized for total read count and feature length. For each feature, the CV across tissues/samples was calculated (Standard deviation/(Mean +1$))$ and ranked.

\section{Weighted gene co-methylation network analysis}

We employed weighted gene co-methylation network analysis [35,50,51] as described in $R$ [52] to find weighted signed co-methylation networks (modules) in the cortex, cerebellum and peripheral blood using log transformed MEDIPS scores. For each genomic feature we performed hierarchical clustering of the samples, based on Pearson correlation, and mapped the final sample dendrogram to three traits (cortex, cerebellum and blood) to describe the sample-trait relationship. For each genomic feature type we performed hierarchical clustering of the topological overlap matrix. Leaves of the tree were grouped into modules, which is a cluster of highly co-methylated genomic locations (GL). After finding the modules, the next step was to describe the relationship between modules and each tissue (that is, cortex, cerebellum and peripheral blood). For this we calculated the Pearson correlation coefficient for the module representative also known as module eigengene (ME), which is the first principal component of each module's methylation profile, and each trait. The GL-trait relation (or gene significance GS if thinking in terms of gene expression) was defined as (the absolute value of) the correlation between the GL methylation profile and the trait. For each module, we also calculated a quantitative measure of module membership as (the absolute value of) the correlation of the ME and the GL methylation profile. This measure allows us to assess the similarity between a module's DNA methylation profile and DNA methylation at a genomic location.

\section{Bisulfite pyrosequencing analysis}

Genomic DNA $(0.5 \mu \mathrm{g})$, extracted from dissected brain samples and blood (Supplementary Table 1 in Additional file 1), was bisulfite converted using the EZ 96DNA methylation kit (Zymo Research, Irvine, CA, USA) following the manufacturer's standard protocol. Fully methylated and unmethylated samples were included throughout the experimental procedure as assay controls. Pyrosequencing assays were designed using the Pyromark Assay Design Software (Qiagen, Crawley, UK). Bisulfite-PCR amplification was performed in duplicate using Hot Star Taq DNA polymerase (Qiagen, UK) and optimized cycling conditions. Pyrosequencing was performed using the Pyromark Q24 (Qiagen, UK). A full list of bisulfite PCR and sequencing primers is given in Supplementary Table 17 in Additional file 1.

\section{Gene expression analysis}

RNA was extracted from matched cerebellum and frontal cortex samples from 42 LBBND donors using the Trizol extraction method and purified using an RNeasy Mini Kit with DNase I digestion (Qiagen, UK) according to the manufacturer's instructions. RNA was tested for purity and degradation using an Agilent 2100 Bioanalyzer and RNA 6000 Nano kit (Agilent Technologies, Wokingham, UK). RNA was biotinylated and amplified using the Illumina TotalPrep ${ }^{\mathrm{TM}}$ RNA Amplification kit (Life Technologies, Paisley, UK). Gene expression was assessed using Illumina HumanHT-12 v4 microarrays (Illumina, San Diego, California, USA) according to the standard manufacturer's protocol. Following scanning, signal intensities for each probe were extracted using Illumina GenomeStudio and imported into $\mathrm{R}$ using the Lumi package within Bioconductor. Probes relating to the top 50 cerebellum-cortex DMRs were tested for expression differences between cerebellum and cortex.

\section{Genotyping arrays and CNV analysis}

For the individuals assessed by MeDIP-seq, Affymetrix SNP 6.0 arrays (Affymetrix, High Wycombe, UK) were used to genotype samples from two tissues according to the manufacturers' standard protocol. CNVs were identified using the PennCNV program [53]. Briefly, this implements a hidden Markov model that integrates multiple sources of information to infer CNV calls for individual genotyped samples. CNVs were stringently called by comparing the duplicate arrays from each individual. 


\section{Data availability}

The methylomic data generated in this project is available for browsing as UCSC Genome Browser tracks from our laboratory website [15]. Raw data have also been deposited into the Human Epigenome Atlas $[16,17]$ and will be integrated into this resource as part of the next data release by NIH Epigenomics Roadmap Initiative.

\section{Additional material}

Additional file 1: Supplementary figures and tables. This file contains Supplementary Figures 1 to 10 and Supplementary Tables 1 to 17.

\begin{abstract}
Abbreviations
BA: Brodmann area; bp: base pair; CDS: coding sequence; CGI: CpG island; CNV: copy number variation; CV: coefficient of variance; DMR: differentially methylated region; GL: genomic location; GS: gene significance; HCP: high CG content promoter; ICP: intermediate CG content promoter; IPA: ingenuity pathway analysis; LCP: Iow CG content promoter; ME: module eigengene; MeDIP-seq: methylated DNA immunoprecipitation combined with ultra-deep sequencing; o/e: observed/expected; PCR: polymerase chain reaction; SNP: single nucleotide polymorphism; TS-DMR: tissue-specific differentially methylated region; UTR: untranslated region.
\end{abstract}

\section{Acknowledgements}

The authors would like to acknowledge Dr Stephen Newhouse for his help with the bioinformatics pipeline and Dr Andrew King for his help in neuropathology. This work was funded by NIH grant AG036039 to JM and by $\mathrm{NIH}$ grant U01DA025956 to AM. RD and AD are supported by the NIHR Biomedical Research Centre for Mental Health at the South London and Maudsley NHS Foundation Trust, Kings College London. RP is supported by a UK Medical Research Council (MRC) PhD studentship.

\section{Author details}

'Institute of Psychiatry, King's College London, De Crespigny Park, London, SE5 8AF, UK. ${ }^{2}$ Department of Twin Research and Genetic Epidemiology, King's College London, Westminster Bridge Road, London, SE1 7EH, UK. ${ }^{3}$ Department of Molecular and Human Genetics, Baylor College of Medicine, One Baylor Plaza, Houston, TX 77030, USA.

\section{Authors' contributions}

$J M$ and LS conceived the experiment and participated in analysis and bioinformatics. SL provided samples. CT dissected post-mortem brain samples. MV, RP, and KL performed laboratory work. MD performed MeDIPseq data analysis and bioinformatics. The NIH Roadmap Epigenomics Data Analysis and Coordination Center (CC, RAH, AM) provided bioinformatics support. $A D$ and $R D$ ran the weighted gene co-methylation network analysis. JM and MD drafted the manuscript. All authors read and approved the final manuscript.

\section{Competing interests}

The authors declare that they have no competing interests.

Received: 30 January 2012 Revised: 21 May 2012

Accepted: 15 June 2012 Published: 15 June 2012

\section{References}

1. Suzuki MM, Bird A: DNA methylation landscapes: provocative insights from epigenomics. Nat Rev Genet 2008, 9:465-476.

2. Ma DK, Marchetto MC, Guo JU, Ming GL, Gage FH, Song H: Epigenetic choreographers of neurogenesis in the adult mammalian brain. Nat Neurosci 2010, 13:1338-1344.

3. Guo JU, Ma DK, Mo H, Ball MP, Jang MH, Bonaquidi MA, Balazer JA, Eaves HL, Xie B, Ford E, Zhang K, Ming GL, Gao Y, Song H: Neuronal activity modifies the DNA methylation landscape in the adult brain. Nat Neurosci 2011, 14:1345-1351.

4. Lubin FD, Roth TL, Sweatt JD: Epigenetic regulation of BDNF gene transcription in the consolidation of fear memory. J Neurosci 2008, 28:10576-10586

5. Renthal W, Nestler EJ: Histone acetylation in drug addiction. Semin Cell Dev Biol 2009, 20:387-394.

6. Migliore L, Coppede F: Genetics, environmental factors and the emerging role of epigenetics in neurodegenerative diseases. Mutat Res 2009, 667:82-97.

7. Nakahata Y, Grimaldi B, Sahar S, Hirayama J, Sassone-Corsi P: Signaling to the circadian clock: plasticity by chromatin remodeling. Curr Opin Cell Biol 2007, 19:230-237.

8. Samaco RC, Neul JL: Complexities of Rett syndrome and MeCP2. J Neurosci 2011, 31:7951-7959.

9. Mill J, Tang T, Kaminsky Z, Khare T, Yazdanpanah S, Bouchard L, Jia P, Assadzadeh A, Flanagan J, Schumacher A, Wang SC, Petronis A: Epigenomic profiling reveals DNA-methylation changes associated with major psychosis. Am J Hum Genet 2008, 82:696-711.

10. Roth RB, Hevezi P, Lee J, Willhite D, Lechner SM, Foster AC, Zlotnik A: Gene expression analyses reveal molecular relationships among 20 regions of the human CNS. Neurogenetics 2006, 7:67-80.

11. Khaitovich P, Muetzel B, She X, Lachmann M, Hellmann I, Dietzsch J, Steigele S, Do HH, Weiss G, Enard W, Heissig F, Arendt T, Nieselt-Struwe K, Eichler EE, Paabo S: Regional patterns of gene expression in human and chimpanzee brains. Genome Res 2004, 14:1462-1473.

12. Johnson MB, Kawasawa YI, Mason CE, Krsnik Z, Coppola G, Bogdanovic D, Geschwind DH, Mane SM, State MW, Sestan N: Functional and evolutionary insights into human brain development through global transcriptome analysis. Neuron 2009, 62:494-509.

13. Ladd-Acosta C, Pevsner J, Sabunciyan S, Yolken RH, Webster MJ, Dinkins T, Callinan PA, Fan JB, Potash JB, Feinberg AP: DNA methylation signatures within the human brain. Am J Hum Genet 2007, 81:1304-1315.

14. Chavez L, Jozefczuk J, Grimm C, Dietrich J, Timmermann B, Lehrach $H_{4}$ Herwig R, Adjaye J: Computational analysis of genome-wide DNA methylation during the differentiation of human embryonic stem cells along the endodermal lineage. Genome Res 2010, 20:1441-1450.

15. KCL Psychiatric Epigenetics Data. [http://epigenetics.iop.kcl.ac.uk/brain].

16. MeDIP-seq data page in Human Epigenome Atlas. [http://genboree.org/ REST/N1/shortUrl/83m7yY?format=redirect].

17. Human Epigenome Atlas. [http://www.epigenomeatlas.org].

18. Bernstein BE, Stamatoyannopoulos JA, Costello JF, Ren B, Milosavljevic A, Meissner A, Kellis M, Marra MA, Beaudet AL, Ecker JR, Farnham PJ, Hirst M, Lander ES, Mikkelsen TS, Thomson JA: The NIH Roadmap Epigenomics Mapping Consortium. Nat Biotechnol 2010, 28:1045-1048.

19. Maunakea AK, Nagarajan RP, Bilenky M, Ballinger TJ, D'Souza C, Fouse SD, Johnson BE, Hong C, Nielsen C, Zhao Y, Turecki G, Delaney A, Varhol R, Thiessen N, Shchors K, Heine VM, Rowitch DH, Xing X, Fiore C, Schillebeeckx M, Jones SJ, Haussler D, Marra MA, Hirst M, Wang T, Costello JF: Conserved role of intragenic DNA methylation in regulating alternative promoters. Nature 2010, 466:253-257.

20. Weber M, Hellmann I, Stadler MB, Ramos L, Paabo S, Rebhan M, Schubeler D: Distribution, silencing potential and evolutionary impact of promoter DNA methylation in the human genome. Nat Genet 2007, 39:457-466.

21. Doi A, Park IH, Wen B, Murakami P, Aryee MJ, Irizarry R, Herb B, LaddAcosta C, Rho J, Loewer S, Miller J, Schlaeger T, Daley GQ, Feinberg AP: Differential methylation of tissue- and cancer-specific CpG island shores distinguishes human induced pluripotent stem cells, embryonic stem cells and fibroblasts. Nat Genet 2009, 41:1350-1353.

22. Sato K, Yabe I, Fukuda Y, Soma H, Nakahara Y, Tsuji S, Sasaki H: Mapping of autosomal dominant cerebellar ataxia without the pathogenic PPP2R2B mutation to the locus for spinocerebellar ataxia 12. Arch Neurol 2010, 67:1257-1262.

23. Couve A, Restituito S, Brandon JM, Charles KJ, Bawagan H, Freeman KB, Pangalos MN, Calver AR, Moss SJ: Marlin-1, a novel RNA-binding protein associates with GABA receptors. J Biol Chem 2004, 279:13934-13943.

24. Baala L, Briault S, Etchevers HC, Laumonnier F, Natiq A, Amiel J, Boddaert N, Picard C, Sbiti A, Asermouh A, Attie-Bitach T, Encha-Razavi F, Munnich A, Sefiani A, Lyonnet S: Homozygous silencing of T-box transcription factor 
EOMES leads to microcephaly with polymicrogyria and corpus callosum agenesis. Nat Genet 2007, 39:454-456.

25. Werner H, Dimou L, Klugmann M, Pfeiffer S, Nave KA: Multiple splice isoforms of proteolipid M6B in neurons and oligodendrocytes. Mol Cell Neurosci 2001, 18:593-605.

26. Makoff A, Lelchuk R, Oxer M, Harrington K, Emson P: Molecular characterization and localization of human metabotropic glutamate receptor type 4. Brain Res Mol Brain Res 1996, 37:239-248.

27. Ramakers GJ, Avci B, van Hulten P, van Ooyen A, van Pelt J, Pool CW, Lequin MB: The role of calcium signaling in early axonal and dendritic morphogenesis of rat cerebral cortex neurons under non-stimulated growth conditions. Brain Res Dev Brain Res 2001, 126:163-172.

28. Toba S, Tenno M, Konishi M, Mikami T, Itoh N, Kurosaka A: Brain-specific expression of a novel human UDP-GalNAc:polypeptide Nacetylgalactosaminyltransferase (GaINAc-T9). Biochim Biophys Acta 2000, 1493:264-268

29. Gibney GT, Zhang JH, Douglas RM, Haddad GG, Xia Y: $\mathrm{Na}(+) / \mathrm{Ca}(2+)$ exchanger expression in the developing rat cortex. Neuroscience 2002, 112:65-73.

30. Zhong $W$, Jiang $M M$, Weinmaster $G$, Jan $L Y$, Jan $Y N$ : Differential expression of mammalian Numb, Numblike and Notch1 suggests distinct roles during mouse cortical neurogenesis. Development 1997, 124:1887-1897.

31. Hayes DM, Braud S, Hurtado DE, McCallum J, Standley S, Isaac JT, Roche KW: Trafficking and surface expression of the glutamate receptor subunit, KA2. Biochem Biophys Res Commun 2003, 310:8-13.

32. Sun Y, Hu J, Zhou L, Pollard SM, Smith A: Interplay between FGF2 and BMP controls the self-renewal, dormancy and differentiation of rat neural stem cells. J Cell Sci 2011, 124:1867-1877.

33. Boulle F, van den Hove DL, Jakob SB, Rutten BP, Hamon M, van Os J, Lesch KP, Lanfumey L, Steinbusch HW, Kenis G: Epigenetic regulation of the BDNF gene: implications for psychiatric disorders. Mol Psychiatry 2011, 17:584-596.

34. Kajiwara Y, Buxbaum JD, Grice DE: SLITRK1 binds 14-3-3 and regulates neurite outgrowth in a phosphorylation-dependent manner. Biol Psychiatry 2009, 66:918-925.

35. Zhang $B$, Horvath S: A general framework for weighted gene coexpression network analysis. Stat Appl Genet Mol Biol 2005, 4:Article17.

36. GeneMANIA. [http://www.genemania.org/].

37. Deaton AM, Webb S, Kerr AR, Illingworth RS, Guy J, Andrews R, Bird A: Cell type-specific DNA methylation at intragenic CpG islands in the immune system. Genome Res 2011, 21:1074-1086.

38. Kim SY, Mo JW, Han S, Choi SY, Han SB, Moon BH, Rhyu IJ, Sun W, Kim H: The expression of non-clustered protocadherins in adult rat hippocampal formation and the connecting brain regions. Neuroscience 2010, 170:189-199.

39. Cosgaya JM, Chan JR, Shooter EM: The neurotrophin receptor p75NTR as a positive modulator of myelination. Science 2002, 298:1245-1248.

40. Bedogni F, Hodge RD, Nelson BR, Frederick EA, Shiba N, Daza RA, Hevner RF: Autism susceptibility candidate 2 (Auts2) encodes a nuclear protein expressed in developing brain regions implicated in autism neuropathology. Gene Expr Patterns 2010, 10:9-15.

41. Herbert MR: SHANK3, the synapse, and autism. N Engl J Med 365:173-175.

42. Whitford KL, Marillat V, Stein E, Goodman CS, Tessier-Lavigne M, Chedotal A, Ghosh A: Regulation of cortical dendrite development by Slit-Robo interactions. Neuron 2002, 33:47-61.

43. Borgel J, Guibert S, Li Y, Chiba H, Schubeler D, Sasaki H, Forne T, Weber M: Targets and dynamics of promoter DNA methylation during early mouse development. Nat Genet 42:1093-1100.

44. Petronis A: Epigenetics as a unifying principle in the aetiology of complex traits and diseases. Nature 2010, 465:721-727.

45. Davies MN, Lawn S, Whatley S, Fernandes C, Williams RW, Schalkwyk LC: To what extent is blood a reasonable surrogate for brain in gene expression studies: estimation from mouse hippocampus and spleen. Front Neurosci 2009, 3:54

46. Rakyan VK, Down TA, Balding DJ, Beck S: Epigenome-wide association studies for common human diseases. Nat Rev Genet 2011, 12:529-541.

47. Schalkwyk LC, Meaburn EL, Smith R, Dempster EL, Jeffries AR, Davies MN, Plomin R, Mill J: Allelic skewing of DNA methylation is widespread across the genome. Am J Hum Genet 2010, 86:196-212.

48. MAQ: Mapping and Assembly with Qualities. [http://maq.sourceforge.net/ ].
49. FastQC. [http://www.bioinformatics.babraham.ac.uk/projects/fastqc/].

50. Horvath S, Dong J: Geometric interpretation of gene coexpression network analysis. PLoS Comput Biol 2008, 4:e1000117.

51. Horvath S, Zhang B, Carlson M, Lu KV, Zhu S, Felciano RM, Laurance MF, Zhao W, Qi S, Chen Z, Lee Y, Scheck AC, Liau LM, Wu H, Geschwind DH, Febbo PG, Kornblum HI, Cloughesy TF, Nelson SF, Mischel PS: Analysis of oncogenic signaling networks in glioblastoma identifies ASPM as a molecular target. Proc Natl Acad Sci USA 2006, 103:17402-17407.

52. Langfelder $\mathrm{P}$, Horvath S: WGCNA: an R package for weighted correlation network analysis. BMC Bioinformatics 2008, 9:559.

53. PennCNV. [http://www.openbioinformatics.org/penncnv/].

doi:10.1186/gb-2012-13-6-r43

Cite this article as: Davies et al:: Functional annotation of the human brain methylome identifies tissue-specific epigenetic variation across brain and blood. Genome Biology 2012 13:R43.

\section{Submit your next manuscript to BioMed Central and take full advantage of:}

- Convenient online submission

- Thorough peer review

- No space constraints or color figure charges

- Immediate publication on acceptance

- Inclusion in PubMed, CAS, Scopus and Google Scholar

- Research which is freely available for redistribution 